\title{
Qber glaziäre Stauchungen in den Mellendorfer und Brelinger Bergen nördlich von Hannover
}

\author{
Von Hans Dietrich Lang, Hannover
}

\author{
Mit 4 Abbildungen im Text
}

Zus a mmenf a s sung: Die Mellendorfer und Brelinger Berge sind ein Teil des großen drenthestadialen Stauch-Endmoränenzuges, der sich von den Niederlanden bis in die Gegend nördlich Braunschweig verfolgen läßt. Die z. T. schon sehr lange bekannten Tonvorkommen bei Mellendorf sind vom Eise verfrachtete Schollen oder Schuppen. Das Einfallen schräggestellter Schichten und das Streichen der Tonschuppen sprechen für einen Eisschub aus ENE, aus W bis WNW und aus N. Das Einfallen schräggestellter Sande und Kiese in den Brelinger Bergen dagegen deutet auf eine Bewegungsrichtung des Eises von $\mathrm{N}$ nach $\mathrm{S}$.

Mikropaläontologische Untersuchungen haben ergeben, daß in den Tonen der Melíendorfer und Brelinger Berge Foraminiferen der Unterkreide ab Hauterive und auch einzelne der Oberkreide vorkommen. Die nachgewiesenen Schubrichtungen weisen in Gebiete, in denen die entsprechenden alten Schichten der Unterkreide anstehen. Danach haben wir mit Transportwegen von maximal $7-8 \mathrm{~km}$ Länge zu rechnen.

Die flachwelligen Mellendorfer Berge sind von einer Geschiebelehmdecke überzogen. Nach der Stauchung ist das Eis über sie hinweg nach $\mathrm{S}$ vorgestoßen. Sie sind also in einer Vorstoßphase des Drenthestadiums entstanden. Die Brelinger Berge, die sehr frische Oberflächenformen zeigen und denen eine Geschiebelehmdecke fehlt, sind jünger und erst in einer späten, einer Rückzugsphase, entstanden. Das Inlandeis ist nicht mehr über sie hinweggegangen. $\mathrm{ab}$.

Insgesamt zeichnen sich im Untersuchungsgebiet 3, vielleicht sogar 4 drenthestadiale Eisvorstöße

Abstract: The Mellendorf and Brelingen hills are part of the large chain of contorted endmoraines (Drenthe stadial) which can be followed from the Netherlands to the region north of Braunschweig. The clay occurrences near Mellendorf, parts of them well-known for a long time, are blocks or overthrusts transported there by glacial movements. The dip of the tilted beds and the strike of clay occurrences indicate glacial thrusts from ENE, from W, WNW and from N. The dip of tilted sands and gravels in the Brelingen hills, however, foints to a direction of ice motion from $\mathrm{N}$ to $\mathrm{S}$.

Micropalaeontological investigations in the clays of the Mellendorf and Brelingen hills have revealed the existence of foraminifera of Lower Cretaceous age, beginning with the Hauterivian, and $\mathrm{a}$ few of them also of the Upper Cretaceous. The proved thrust directions point to regions where the respective old beds of the Lower Cretaceous are exposed. Accordingly the length of transport must have been about $7-8 \mathrm{~km}$.

The slightly undulating country around Mellendorf is covered by a sheet of till. After the contortion, the ice advanced over the Mellendorf hills in a southern direction. The Brelingen hills, showing very recent relief and without a covering till, are younger and belong to a later recessional phase; the ice did not override them.

The investigations demonstrate that three, perhaps even four, glacial thrusts of the Drenthe stadial took part in the genesis of this region.

\section{Einführung}

Seit über 120 Jahren sind in den etwa $20-25 \mathrm{~km}$ nördlich Hannover gelegenen Mellendorfer und Brelinger Bergen Vorkommen von Tonen der Unterkreide, besonders des Barrême, bekannt. F. A. Roemer (1841), Credner (1865), G. Müller (1893), v. Koenen (1902), Forche (1949) und Bartenstein (1959) berichteten darüber. Forche (1949) konnte nachweisen, daß es sich bei diesen bisher als autochthon angesehenen Vorkommen um vom Eise verschleppte Schollen handelt, als deren Herkunftsgebiet er den Salzstock von Hope vermutete.

ForChe (1949) und BarTenstein (1959) bezeichneten die Barrême-Vorkommen in der Umgebung von Mellendorf als Schollen. In Anlehnung an eine von VIETE (1960) vorgeschlagene Definition werden sie hier, abgesehen von den Vorkommen bei Bennemühlen, 
als Schuppen bezeichnet, da sie meist in mehrfacher Wiederholung auftreten und über ein größeres Gebiet ein gleichgerichtetes Streichen zeigen (LANG 1963).

Die bisherige Darstellung dieses Gebietes auf geologischen Übersichtskarten (z. B. Geotektonische Karte von Nordwestdeutschland im Maßstab 1:100 000, Aldinger, Closs, SchotT \& WAGER 1946) beruht weitgehend auf einer von Beschoren (1933) durchgeführten und nicht veröffentlichten Übersichtskartierung des Blattes 3424 (1815) Mellendorf der top. Karte $1: 25000$.

Die in der vorliegenden Arbeit angeführten mikropaläontologischen Untersuchungen wurden freundlicherweise von den Herren Dr. Hiltermann und Koch von der Bundesanstalt für Bodenforschung in Hannover durchgeführt. Ihnen sei an dieser Stelle nochmals dafür gedankt.

\section{Gang der Untersuchungen und Hilfsmittel}

Im Rahmen einer geologischen Spezialkartierung des Blattes 3424 Mellendorf der Karte 1:25000 hatte der Autor Gelegenheit, das Gebiet der Mellendorfer und Brelinger Berge eingehend geologisch zu bearbeiten. Besonderes Augenmerk galt dabei den glaziärtektonischen Vorgängen, der Größe und Ausdehnung der Tonvorkommen, ihrer stratigraphischen Stellung, ihrer Transportrichtung und ihrer Herkunft. Das gesamte Untersuchungsgebiet wurde mit einem zum Teil sehr dichten Netz von 2-m-Bohrungen überzogen, wobei eine Vielzahl neuer, bis dahin unbekannter Tonvorkommen nachgewiesen werden konnte, die teils direkt an der Oberfläche anstehen, teils unter geringmächtiger Überdeckung liegen.

Als wertvolle Hinweise auf Tonvorkommen erwiesen sich neben den im Gelände beobachteten Feuchtigkeits- und Vegetationsverhältnissen alte Karten und Luftbilder.

Die neueste Ausgabe der top. Karte $1: 25$ 000, B1. 3424 Mellendorf, enthält, verglichen mit älteren Ausgaben, z. B. der von 1899, nur noch wenige Hinweise auf Tonvorkommen (Bartenstein 1959). Von den früher in Scherenbostel, Wiechendorf und Mellendorf vorhandenen und in dieser Karte verzeichneten Ziegeleien besteht keine mehr. Da auch der Abbau von Mergeln zu Düngezwecken schon lange der Vergangenheit angehört, sind sämtliche Ton- und Mergelgruben verfallen, teilweise oder ganz zugeschüttet oder stehen voller Wasser. Eine Reihe von ihnen ist in den neueren Ausgaben dieser Karte auch nicht mehr verzeichnet. Auch die vielen kleinen, hie und da im Walde gelegenen und auf keiner Karte verzeichneten Ton- oder Mergelgruben sind weitgehend zugewachsen.

Ausgehend von diesen alten Aufschlüssen war es möglich, eine Reihe von Tonvorkommen über einige hundert Meter zu verfolgen, manche sogar über $1 \mathrm{~km}$ weit. Ein sicherer Hinweis auf Tonvorkommen waren Schlaglöcher und sumpfige Stellen auf Waldwegen, Pfützen im Wald, besonders im zeitigen Frühjahr und nach längeren Niederschlagsperioden, und das Auftreten feuchtigkeitsliebender Bäume und Sträucher inmitten des sonst hier vorherrschenden Nadelwaldes. Auch bei einer Überdeckung bis zu $2 \mathrm{~m}$ waren die veränderten Feuchtigkeitsverhältnisse, besonders bei den breiteren Tonschuppen, noch gut zu erkennen.

Im frühen Frühjahr bzw. im Spätherbst zeichneten sich auf den kahlen Feldern oft Streifen auffallend dichter Geröllbestreuung ab, die sich im Anschluß an Aufschlüsse meist als Ausstriche schläggestellter Kieslagen erwiesen.

Einigen Aufschluß über das Vorhandensein von Tonen geben uns Luftbilder, besonders dann, wenn sie im späten Herbst oder im zeitigen Frühjahr vor dem Beginn der Vegetatationsperiode aufgenommen wurden. Die Gebiete, in denen Wasser über undurchlässigen Tonen gestaut wird, heben sich durch ihre dunklere Farbe von der meist helleren, sandigen Umgebung ab. Am deutlichsten sind diese Helligkeitsunterschiede auf Ackerflächen zu erkennen. Auf Grünland sind sie schon weniger deutlich, und in Waldgebieten werden sie von Bäumen und Sträuchern verdeckt. Wenn eine Tonschuppe eine Mindestbreite unterschreitet, wirkt sie sich auch nicht mehr als "Wasserstauer" aus und ist an der Oberfläche nicht mehr auszumachen. 


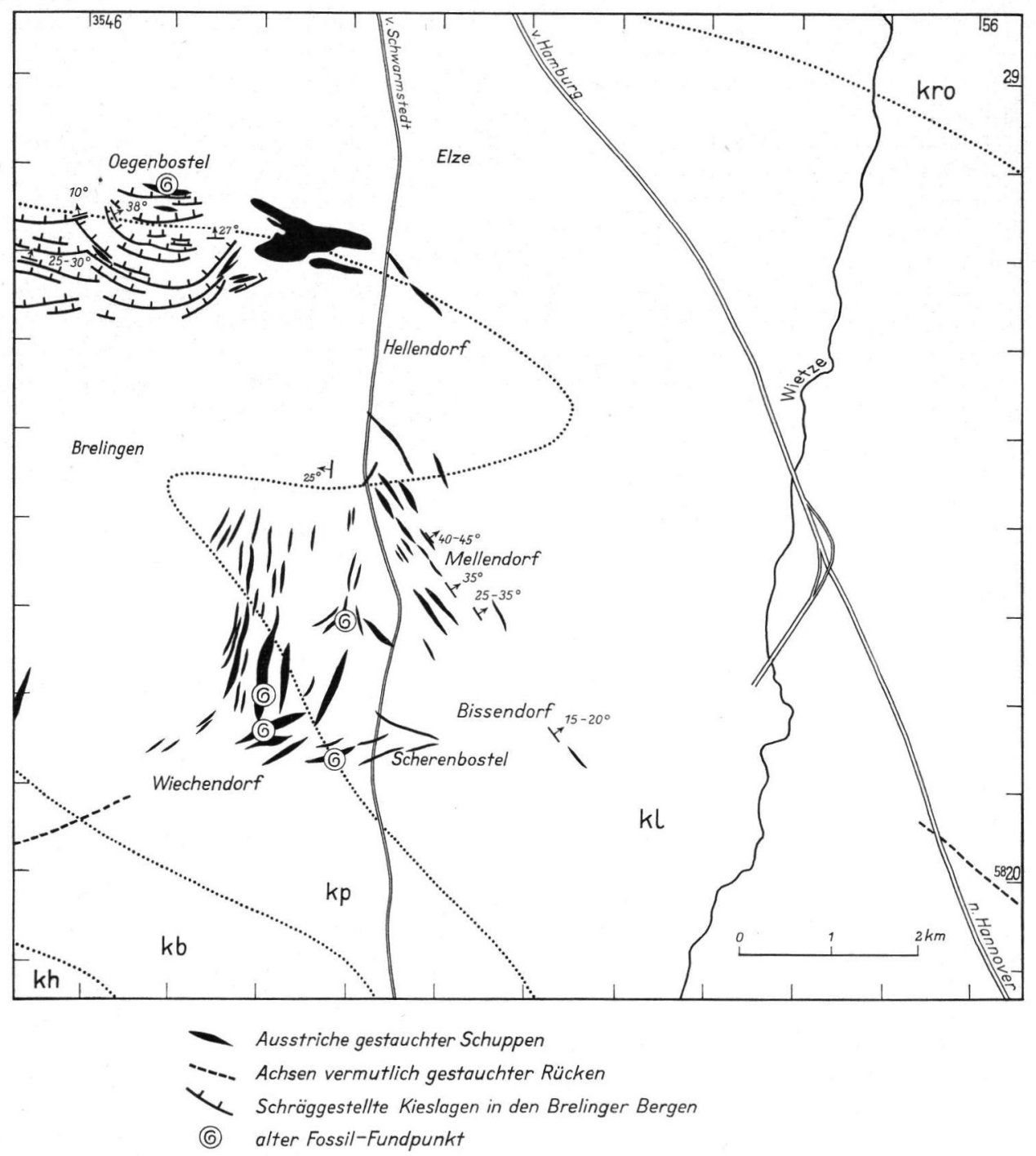

Abb. 1. Vom Inlandeis verfrachtete Tonschollen bzw. -schuppen und schräggestellte Sande und Kiese in den Mellendorfer und Brelinger Bergen.

\section{Oberflächenform und geologischer Aufbau}

\subsection{Die Mellendorfer Berge und ihre Umgebung}

Als die Mellendorfer Berge bezeichnen wir das hügelige und weitgehend bewaldete Gebiet westlich der Wietze-Niederung ( $+40 \mathrm{~m} \mathrm{NN}$ ) zwischen den Ortschaften Mellendorf, Brelingen, Wiechendorf und Bissendorf. Sie erreichen im Witrem-Berg eine Höhe von fast $90 \mathrm{~m}$ NN, erheben sich also um etwa $50 \mathrm{~m}$ über die Wietze-Niederung. SPEthmann (1908) und BEERHENKE (1946) deuteten sie nach den Oberflächenformen als einen Endmoränenzug.

Abgesehen von den schon erwähnten Tonvorkommen bestehen sie in der Hauptsache aus saalezeitlichen Sanden und Kiesen, die nach den vorliegenden Bohrungen eine Mächtigkeit von 30-50 m erreichen können. Darunter folgen Tone der höheren Unterkreide, die 
leicht nach NE einfallen. Inwieweit an ihrem Aufbau noch ältere pleistozäne Schichten beteiligt sind, ist nicht bekannt. Eine Decke von Geschiebelehm oder bzw. und Geschiebedecksand überzieht weite Teile der Mellendorfer Berge und bedingt flachwellige Geländeformen.

Besonders dort, wo Geschiebelehm und Geschiebedecksand fehlen oder nur eine geringe Mächtigkeit haben, sind Vorkommen von dunkelgrauen bis blaugrauen, sehr zähen Tonen bekannt. Vielfach sind sie mit pleistozänen Sanden verunreinigt und bis in Tiefen um 1,5 m entkalkt. Besonders im Gebiet des heutigen Löns-Sees scheinen sie reich an Toneisenstein-Geoden zu sein.

Auf Abb. 1 sind sämtliche bisher bekannten Ausbisse von Tonvorkommen kartenmäßig dargestellt. Dabei überrascht es zu sehen, wie genau sich die glazigenen Schuppen in nur wenige Richtungen einordnen, wie weit diese Richtungen durchhalten und wie sauber hintereinander gestaffelt die Schuppen auftreten. In der Umgebung von Mellendorf und zwischen Mellendorf und Bissendorf herrscht nordwestliches bis nordnordwestliches Streichen vor, im Gebiet von Scherenbostel etwa west-östliches und bei Wiechendorf und im Gebiet des Löns-Sees fast genau nord-südliches.

Uber den inneren Aufbau der glazigenen Schuppen ist nur wenig bekannt. Die verfallenen und verwachsenen Aufschlüsse lassen heute keine Einzelheiten mehr erkennen. Credner (1865) schreibt dazu (S. 249, Abschnitt 6): „Eine Beobachtung über sein (des Tones) Einfallen ist am Ausgehenden der jedenfalls sehr flach gelagerten Schichten nicht möglich." Hingegen schreibt Bartenstern (1959, S. 456), daß in der nahe dem Löns-See niedergebrachten Bohrung SB. 89 die zwischen 14 und $25 \mathrm{~m}$ Tiefe erbohrten Tone der Unterkreide z. T. senkrecht stehen. Aus den allerdings sehr ungenauen Schilderungen älterer Einwohner geht übereinstimmend hervor, daß die Aufschlüsse meist schmal und tief waren und in den verschiedensten Gruben, auch in tieferen Baugruben, der blaugraue Ton plötzlich mit scharfer Grenze an den Sand stieß und daß die ehemalige große und sehr tiefe Tongrube der Ziegelei auf dem Witrem-Berg, der heutige Löns-See, angeblich in einer Nacht voll Wasser gelaufen sei, ohne daß man das darin befindliche Gerät habe retten können.

In einer Reihe von Sand- und Kiesgruben in der Umgebung von Mellendorf und in Kanalisationsgräben in Mellendorf und Bissendorf waren bzw. sind vom Eise schräggestellte Kiese, Sande und Schluffe aufgeschlossen. Manche dieser Lagen ließen sich, vom Aufschluß ausgehend, an der Oberfläche verfolgen. In der alten Sandgrube auf dem OrtBerg zwischen Wennebostel und Mellendorf sind sowohl an der N-als auch an der S-Wand schräggestellte Sande und Schluffe aufgeschlossen, die mit $25-35^{\circ}$ nach ENE einfallen. In gleicher Richtung fielen Schichten ein, die im Sommer 1963 zeitweilig in einer Baugrube in Bissendorf, an der nach Wennebostel führenden Straße, aufgeschlossen waren. Das Einfallen betrug hier $15-20^{\circ}$. Besonders eindrucksvoll war ein Aufschluß im Gebiet der alten Sandgruben am S-Rande des Ortes Mellendorf, etwa $400 \mathrm{~m}$ westlich des Wohlen-Berges (Abb. 2). Im südlichen Teil des ausgedehnten Grubengeländes war im Sommer 1963 die Aufschiebungsfläche eines tonigen Geschiebelehmes auf drenthestadiale Verschüttsande aufgeschlossen, vor der die Sandschichten aufgerichtet und zum Teil schon leicht nach W überkippt waren. Die schaufelförmige Aufschiebungsfläche fiel flach nach ENE ein.

Ein von den bisherigen Richtungen abweichendes Einfallen zeigen Sande und Kiese in der Sandgrube der Firma Franke auf dem Kickens-Berg nordwestlich Mellendorf. Die Schichten fallen mit durchschnittlich $25^{\circ}$ nach W ein. Besonders in dieser Grube treten sporadisch zwischen Sand- und Kieslagen Fetzen oder Schuppen von unreinen grauen Tonen oder von Geschiebelehm auf, die den Abbau sehr erschweren. Auch diese Ton- und Lehmfetzen weisen auf ein Einfallen nach W hin und zeigen zudem, wie intensiv das Eis die ursprünglichen Lagerungsverhältnisse gestört hat.

Diese Beobachtungen fügen sich sehr gut in das bisherige Bild ein und weisen auf einen Eisschub aus W, aus $\mathrm{N}$ und aus ENE. Nach den Versuchen von Köster (1957a, 1957b, 


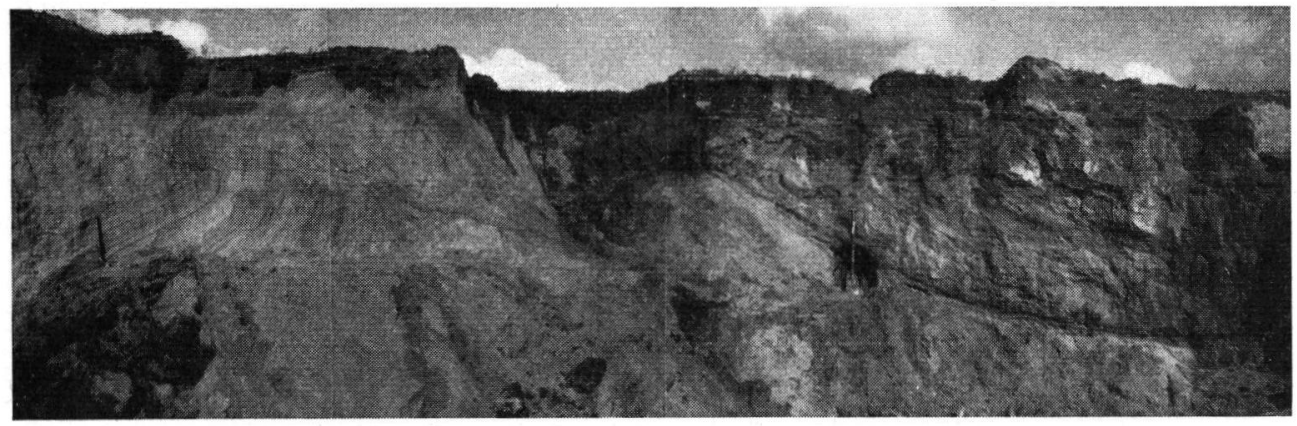

Abb. 2. Sandgrube am Südrande von Mellendorf (Bl. 3424 Mellendorf). Von ENE (rechts) her auf drenthestadiale Verschüttsande aufgeschobener tonreicher Geschiebelehm. Die Sande im linken Teil des Bildes (WSW) sind aufgerichtet und z. T. schon leicht nach WSW überkippt.

1958) und den bisherigen Beobachtungen (KELLER 1954, VIETE 1960) ist diese Bewegungsrichtung des Eises auch aus der Form und dem Streichen der Tonschuppen zu folgern.

Wie auf Abb. $1 \mathrm{zu}$ erkennen ist, biegen die in der Umgebung des Löns-Sees und im Waldgebiet östlich Brelingen N-S-streichenden Stauchschuppen nördlich Wiechendorf nach SW $\mathrm{ab}$ und finden ihre Fortsetzung in einem langgestreckten Rücken, der sich auf Blatt 3424 Otternhagen der top. Karte 1:25000 bis etwa $2 \mathrm{~km}$ westlich Resse verfolgen läßt. Im Bereiche des Blattes Mellendorf treten in Verbindung mit diesem Rücken neben saalezeitlichen Ablagerungen dunkel-braungrauer Geschiebelehm bzw. Geschiebemergel, der wahrscheinlich elstereiszeitlichen Alters ist (LANG 1962), weißgraue, sehr saubere Feinsande, graugrüne, schluffige Feinsande und ein glimmerreicher dunkel-graubrauner Schluff auf. Diese Sande und Schluffe treten nach den bisherigen Beobachtungen nur in Verbindung mit dem wahrscheinlich elstereiszeitlichen Geschiebelehm auf.

Dieser leicht nach S ausgebogene Rücken läuft genau parallel zu weiter im S gelegenen, denen von Frielingen-Heitlingen, von Horst-Engelbostel und von Garbsen (B1. 3523 Stöcken der top. Karte 1 : 25 000). KELler (1955) deutete diese Rücken als „fluvioglaziale“ Bildungen, und zwar als Kames mit Aufpressungserscheinungen. Vor ihm hatte $\mathrm{H}_{\text {AMM }}$ (1929) den südlichsten dieser Rücken als Os angesprochen. Die Kartierarbeiten während der letzten Jahre auf den Blättern 3523, Stöcken, und 3524, Isernhagen, der Karte 1:25000 (Grahle \& Groba 1961 unveröffentl., Lang 1962) zeigten, daß es sich hier wahrscheinlich um glazigen gestauchte Rücken und nicht um „fluvioglaziale“ Bildungen handelt. Ḧ̈LBICH (1962) konnte auf Grund gefügekundlicher Untersuchungen im Gebiet nördlich von Hannover nachweisen, daß diese Rücken vor der Stirn eines zeitweilig stagnierenden Gletschers zusammengeschoben wurden. Diese Ergebnisse decken sich mit denen der Kartierung auf Bl. Mellendorf.

Auch nach SE scheinen sich die Stauchschuppen fortzusetzen. In der SE-Ecke des Blattes 3424 Mellendorf der top. Karte 1:25000, in der Gegend nordwestlich von Großburgwedel, treten neben saalezeitlichen Ablagerungen in verhältnismäßig weiter Verbreitung braungrauer, wahrscheinlich elstereiszeitlicher Geschiebelehm, graugrüner schluffiger Feinsand und dunkelbrauner, glimmerreicher Schluff auf, wie er auch schon im Gebiet des nach SW ziehenden Rückens beobachtet wurde. Diese Ablagerungen scheinen wieder in bestimmten, südöstlich streichenden Streifen angeordnet zu sein. Óstlich Großburgwedel treten dann lange, annähernd westöstlich ziehende Rücken auf (Grahle \& Groba 1961 unveröffentl.). Die räumliche Verteilung der Feinsande und glimmerreichen Schluffe spricht nach den bisherigen Erfahrungen wieder für Stauchungen. Leider ist dieses sehr ebene Gebiet bar jeglicher Aufschlüsse. Auch morphologische Hinweise auf Schuppenstrukturen sind hier nicht vorhanden, sondern treten erst wieder östlich Großburgwedel auf. 
Abgesehen von den Tonvorkommen bei Scherenbostel bilden die glazigenen Stauchschuppen in der weiteren Umgebung von Mellendorf zwei große, nach SE bzw. SW konvexe Bögen, die nördlich Mellendorf zusammenstoßen, sich aber auch etwas zu überschneiden scheinen. Aus dieser bogenförmigen Anordnung und aus dem beobachteten Einfallen von Schluffen, Sanden und Kiesen, an einer Stelle sogar einer Aufschiebungsbahn, lassen sich zwei Gletscher-Loben ableiten. Vor ihrer etwa halbkreisförmigen Stirn wurden bei mehrfachem Oszillieren um eine Mittellage Schichten aus dem Untergrund abgeschert und mit pleistozänen Schluffen, Sanden und Kiesen zusammen verschuppt. Entsprechend der etwa halbkreisförmigen Stirn des Gletschers schwanken auch die Einfallsrichtungen der schräggestellten Schichten wie der Schubbahnen. Wie weit sich die beiden Loben nach W bzw. nach $\mathrm{E}$ ausdehnten, wissen wir bis jetzt noch nicht genau. Einen Uberblick über den Verlauf der nachgewiesenen und vermuteten Stauchungen im Gebiet nördlich Hannover zeigt die Abb. 3.

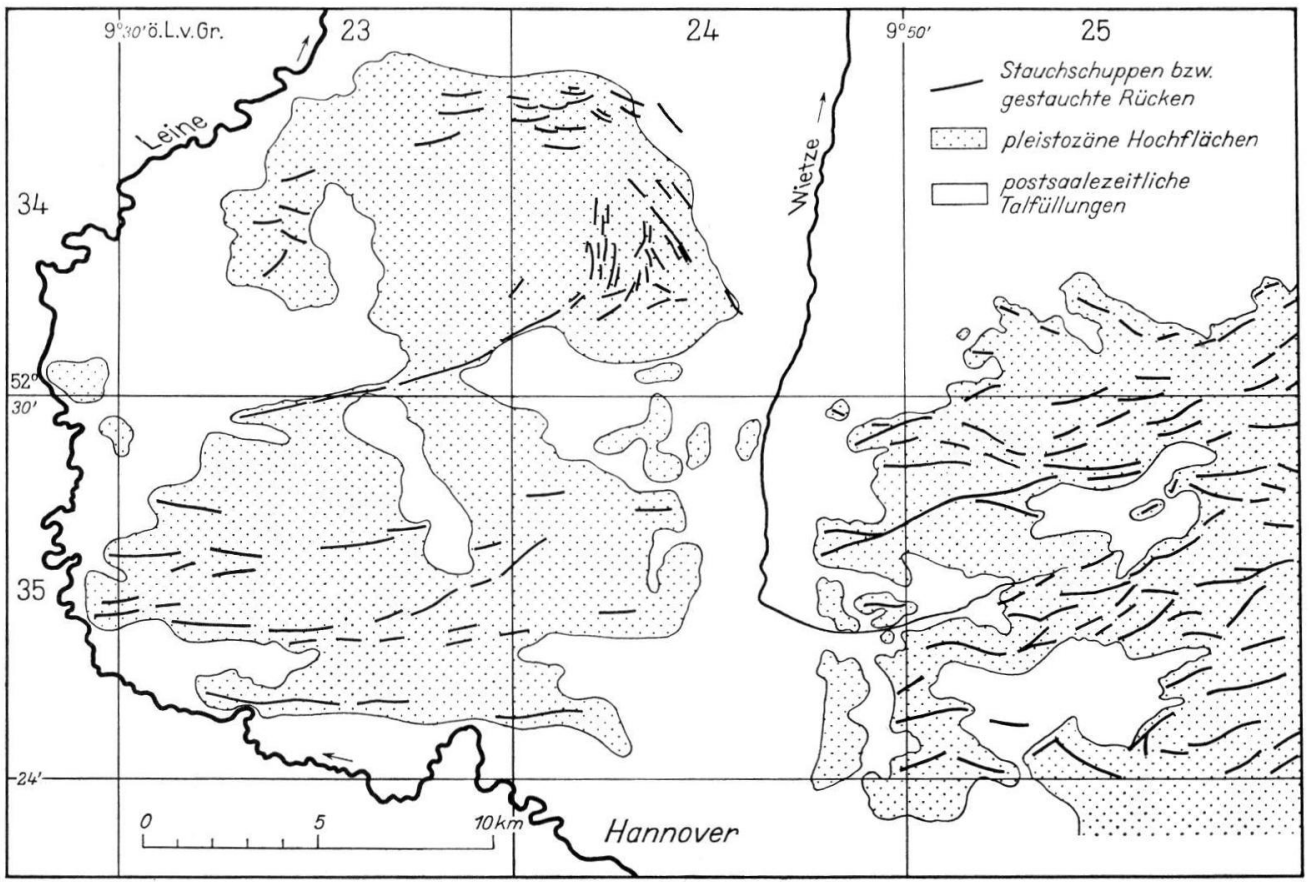

Abb. 3. Glazigene Schuppungen und Stauchungen in der nördlichen Nachbarschaft von Hannover (nach Untersuchungen von H. O. Grahle, E. Groba und H. D. Lang).

Zusammenhänge zwischen der Oberflächenform und dem Schuppenbau sind in den Mellendorfer Bergen zwar vorhanden, aber nur undeutlich ausgebildet. Ihre Form als Ganzes zeigt zwar Übereinstimmung mit den vorherrschenden glaziärtektonischen Richtungen. Die einzelnen Tonvorkommen jedoch heben sich morphologisch nicht heraus. Sie werden von den vorwiegend E-W-verlaufenden Bachtälern vielfach senkrecht geschnitten, und die Rücken zwischen den einzelnen Bachtälern zeigen keinerlei Beziehungen zu den glazialtektonischen Erscheinungen.

\subsection{Die Brelinger Berge}

Im Gegensatz zu den heute eingeebneten, flachen Oberflächenformen in den Mellendorfer Bergen weisen die Brelinger Berge auffallend lebhafte, frische Formen auf, ein Gegensatz, auf den schon Beerhenke (1947) hinwies. Während in den Mellendorfer Ber- 
gen eine beherrschende Vorzugsrichtung nur grob angedeutet ist und eine morphologische Feingliederung fehlt, zeigt bereits die topographische Karte, daß die Brelinger Berge in eine Reihe ost-westlich gestreckter Rücken gegliedert sind. Weit deutlicher noch tritt dieser Befund in Luftbildern hervor, besonders bei stereoskopischer Betrachtung. Es zeigt sich eine erstaunliche Ähnlichkeit mit rezenten Moränenwällen, wie sie z. B. Gripp (1929) aus Spitzbergen abgebildet hat.

Die Brelinger Berge bestehen aus einer Reihe leicht nach $S$ ausgebogener Rücken, die mehrfach hintereinander gestaffelt sind und sich in westöstlicher Richtung girlandenartig aneinanderreihen. Ihr Nordrand ist auffallend steil. Nach S gehen sie ohne scharfe Grenze in eine Geschiebelehmhochfläche über. Vor dem steilen N-Hang hat sich in jüngerer Zeit ein breiter Schuttfuß gebildet. Besonders zwischen den nördlichsten der bogenförmigen Rücken treten steile, meist asymmetrische Täler, in der Regel Trockentäler (BEERENKE 1947), auf.

Schon G. Müller (1893) beschrieb vom N-Hang der Brelinger Berge bei Oegenbostel ein Vorkommen von grauen Tonen mit Fossilien des Alb; auch BESCHOREN (1933) erwähnte ein Vorkommen. Weitere liegen am E-Ende der Brelinger Berge, z. B. am E-Hang des Klages-Berges, wo neben einer alten Mergelgrube eine kleine Quelle auf Tone hinweist. Sie wurden auch im Handbohrer erfaßt (s. Abb. 1). Verglichen mit den Mellendorfer Bergen spielen die Tonvorkommen in den Brelinger Bergen nur eine geringe Rolle. Ihre Anzahl ist gering und ihre Ausdehnung keinesfalls mit der der schon beschriebenen Vorkommen $\mathrm{zu}$ vergleichen.

Unklar ist die Stellung der ausgedehnten Tonvorkommen im Ort Bennemühlen und östlich davon, im Gebiet des Hühnen-Berges. Obwohl sie etwa im Streichen der Brelinger Berge liegen, ist es nach dem morphologischen Bild wenig wahrscheinlich, daß sie dazu gehören. Abgesehen von einer alten, verfallenen Mergelgrube am NW-Rand von Bennemühlen gibt es hier keine nennenswerten Aufschlüsse. Besonders das Vorkommen in Bennemühlen sieht in seiner äußeren Form mehr einer Scholle als einer Schupve ähnlich. Eine genetische Zuordnung zu den Tonen in den Mellendorfer Bergen erscheint eher vertretbar.

Die Kämme der einzelnen Rücken in den Brelinger Bergen werden stets von Kiesen gebildet, während an den S-Hängen und in den Senken feine Sande anstehen. Genau wie die Täler haben die Rücken asymmetrische Form, einen steilen S-Hang und einen flachen N-Hang.

Die nach diesem morphologischen Bild schon zu erwartenden, nach $\mathrm{N}$ einfallenden Schichten sind in verschiedenen Sand- und Kiesgruben in den Brelinger Bergen bzw. an deren N-Hang aufgeschlossen, wie beispielsweise in Sandgrube ScHLÜTER, $500 \mathrm{~m}$ südlich Bestenbostel am N-Hang der Brelinger Berge gelegen. Sande, Kiese, an einer Stelle auch ein anscheinend gebankter Geschiebelehm, fallen mit $25-30^{\circ}$ fast genau nach $\mathrm{N}$ ein.

An der östlichen Abbauwand einer alten, kleinen Kiesgrube in den Brelinger Bergen, etwa $2 \mathrm{~km}$ südwestlich Oegenbostel, fallen die Schichten mit $25-30^{\circ}$ nach $\mathrm{N}$ ein (s. auch Abb. 1). In zwei weiteren kleinen Aufschlüssen wurde ein Einfallen von $10^{\circ} \mathrm{bzw} .38^{\circ}$ nach $\mathrm{N}$ oder senkrecht zum Streichen von Kiesrippen gemessen (s. Abb. 1). Die Asymmetrie der Täler (BeERHenke 1947) ist hier also rein geologisch bedingt.

Nach den bisherigen Untersuchungen tritt in den Brelinger Bergen selbst kein Geschiebelehm in deckenhafter Verbreitung auf. Er setzt, von einigen verschuppten Fetzen abgesehen, erst weiter südlich ein. Im Gegensatz zu den Mellendorfer Bergen sind die Brelinger Berge nach ihrer Aufrichtung nicht mehr vom Inlandeis überfahren worden und haben daher die Frische ihrer Formen, wenn auch durch spätere Vorgänge im periglazialen Klimabereich etwas verwischt, bewahren können.

Die Ergebnisse der geologischen Kartierung decken sich mit den in der Hauptsache auf morphologischen Befunden beruhenden Deutung der Brelinger Berge als Stauchendmoräne (Spethmann 1908, Beerhenke 1947, Woldstedt 1928, 1954). Sie verdanken ihre Entstehung einem Gletscher, der, um eine mittlere Lage oszillierend, mehrfach leicht nach $S$ 
vorstieß und vor seiner Stirne Sand, Kies und Geschiebelehm zusammenschob. Anschließend zog er sich, ohne weiter nach $\mathrm{S}$ vorgestoßen zu sein, nach $\mathrm{N}$ zurück. Im Gegensatz zu den während einer Vorstoßphase entstandenen Mellendorfer Bergen sind die Brelinger Berge die Bildung einer Rückzugsphase (RIChter, SChneider \& W WGer 1951, Woldstedt 1938, KELLER 1953) und erst in einem sehr späten Abschnitt des Drenthe-Stadiums entstanden.

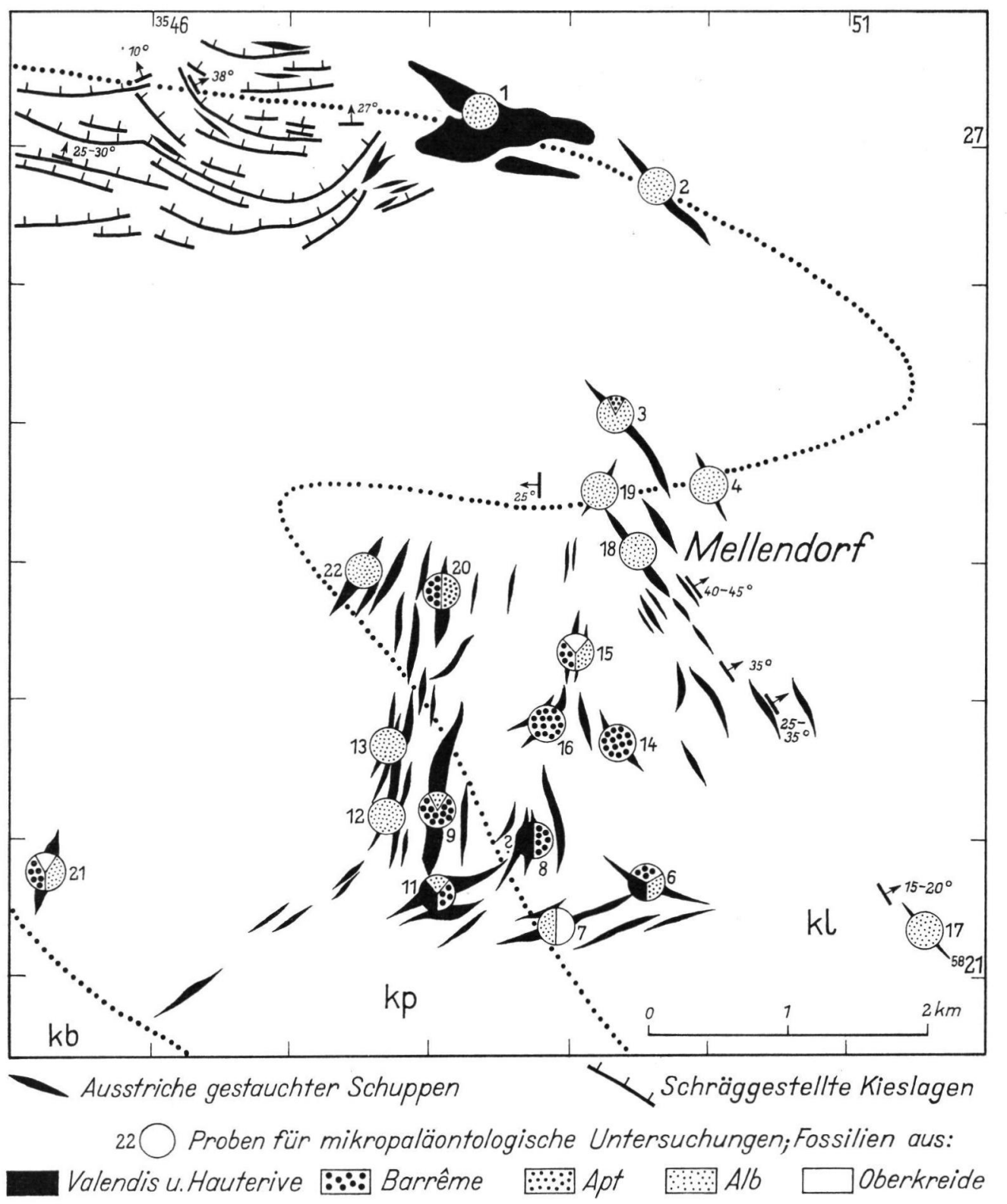

Abb. 4. Die Ergebnisse der mikropaläontologischen Untersuchungen an gestauchten bzw. verschuppten Tonen in den Mellendorfer und Brelinger Bergen. 


\section{Herkunft der Tonschuppen bzw. -schollen}

Im Rahmen der Kartierung wurden 24 Tonproben aus den verschiedenen Vorkommen mikropaläontologisch untersucht. Da Aufschlüsse entweder nicht vorhanden oder nicht mehr zugänglich waren, wurden die Proben mit einer Ausnahme mit einem Handbohrer aus durchschnittlich $1,5 \mathrm{~m}$ Tiefe entnommen. Eine pleistozäne Verunreinigung ist dadurch möglich. Die Ergebnisse dieser Untersuchungen sind auf Tab. 1 und Abb. 4 wiedergegeben.

Entsprechend der Genese der Tonvorkommen, - sie sind vom Eise umgelagert - wurden in manchen der Proben Mikrofossilien verschiedener Stufen der Unterkreide nebeneinander nachgewiesen. Daneben traten auch einzelne Formen der Oberkreide auf. Insgesamt fanden sich Mikrofossilien der Unterkreide ab Hauterive, vielleicht auch des Valendis.

Tabelle 1

Ergebnisse der mikropaläontologischen Untersuchung von Tonproben aus der Umgebung von Mellendorf (Bl. 3424 Mellendorf der top. Karte $1: 25000$ ).

Untersucht von H. HiltermanN und W. Koch.

\begin{tabular}{lccc}
\hline Lfd. & Labor- & Entnahmepunkt und & Mikropaläontologischer \\
Nr. & Nr. & Tiefe & Befund \\
\hline
\end{tabular}

140250 A2/40. Alte Mergelgrube $400 \mathrm{~m}$ NW Bennemühlen, aus $1,5 \mathrm{~m}$ Tiefe. R $3548220 \quad$ H 5827320

240251 A2/126. Am Südrand des Hühnen-Berges b. Elze, aus $1,5 \mathrm{~m}$ Tiefe. R 3549490 H 5826950

340252 A2/130. Alte Tongrube $\mathrm{N}$ des Siemsberges b. Mellendorf, aus $1,5 \mathrm{~m}$ Tiefe. R $3549330 \quad$ H 5825150

$4 \quad 40253 \quad$ A2/182. Alte Sandgrube $750 \mathrm{~m}$ E des Siemsberges $\mathrm{b}$. Mellendorf, aus $1,5 \mathrm{~m}$ Tiefe. R 3549950 H 5824250

540254 A2/174. Alte Tongrube bei der Schwanenburg in Mellendorf, aus $1,5 \mathrm{~m}$ Tiefe. R $3549340 \quad$ H 5824340

640255 B2/388. Scherenbostel, $200 \mathrm{~m}$ E der Bundesstraße, aus $1,5 \mathrm{~m}$ Tiefe. R $3549530 \quad$ H 5821740

740256 B2/28. Alte Ziegelei-Tongrube Scherenbostel, $1,5 \mathrm{~m}$ Tiefe unter Grubensohle. R 3548760 H 5821320

$8 \quad 40257 \quad$ Alte Tongrube $750 \mathrm{~m}$ E des Löns-Sees, aus $1,5 \mathrm{~m}$ Tiefe.

R $3548720 \quad$ H 5822040

940258 Wasserriß am NW-Ende des Löns-Sees, aus $1,5 \mathrm{~m}$ Tiefe.

R $3548080 \quad$ H 5822270

1040259 NW-Ende des Löns-Sees, etwa aus $2 \mathrm{~m}$ Tiefe.

R $3548020 \quad$ H 5822250
Pleistozän mit vereinz. Mikrofossilien des mittleren und unteren Alb.

Unter-Alb, mit Pleistozän verunreinigt.

Unter-Alb, mit Pleistozän (und Ober-Apt) verunreinigt.

Unter-Alb, mit Pleistozän verunreinigt.

Unter-Alb, mit Pleistozän verunreinigt.

Mischfauna aus Mikrofossilien des Unter-Alb, $\mathrm{M}$ i $\neq \mathrm{t}$ e $1-\mathrm{B}$ a $\mathrm{r}$ r êm e, Ober-Hauterive (u. Valendis?).

Im Rückstand vereinzelt Mikrofossilien aus Alb und Oberkreide.

Im Rückstand Mikrofossilien aus dem (mittleren) Barrême (und Hauterive ?); stark durchsetzt mit Gips-Kristallen.

Unteres Barrême, mit Pleistozän verunreinigt. Außerdem einzelne Foraminiferen des Alb.

Vereinzelte Foraminiferen deuten auf Barrême oder Unter-Alb. Daneben einige Radiolarien, die dem Ober-Alb entstammen dürften. 
Lfd. Labor-

Nr. Nr.
Entnahmepunkt und

Tiefe
Mikropaläontologischer

Befund
$1140260 \quad B 2 / 12$. Alte Tongrube NE Wiechendorf, aus $1,5 \mathrm{~m}$ Tiefe.

R 3548040 H 5821610

1240261 B1/9. $400 \mathrm{~m} \mathrm{NW}$ des Löns-Sees, aus $1,5 \mathrm{~m}$ Tiefe.

R $3547750 \quad$ H 5822450

1340262 B1/23. $500 \mathrm{~m} \mathrm{NW}$ des Löns-Sees, aus $1,5 \mathrm{~m}$ Tiefe.

R 3547680 H 5822650

$14 \quad 40263 \quad$ NW-Ende der Tongrube am Osthang des Witrem-Berges, aus 1,5 $\mathrm{m}$ Tiefe. R 3549300 H 5822780

1540264 B2/492. $300 \mathrm{~m} \mathrm{NE}$ des Fernmeldeturmes b. Mellendorf, aus $1,5 \mathrm{~m}$ Tiefe.

R $3549080 \quad$ H 5823410

1640265 B2/179. SW-Ende der Tongrube auf dem Witrem-Berg, aus $1,5 \mathrm{~m}$ Tiefe.

R $3548810 \quad$ H 5822860

$1740266 \mathrm{~B} 3 / 28$. Tonfetzen im Geschiebelehm in einer Baugrube in Bissendorf, 1-1,4 $\mathrm{m}$ Tiefe. R $3551210 \quad$ H 5821620

40267 A2/175. Kanalisationsgraben in Mellendorf, aus $1,5 \mathrm{~m}$ Tiefe.

R $3549420 \quad$ H 5824440

40268 Sandgrube KüKER auf dem Siemsberg in Mellendorf. Probe auf der Grubensohle entnommen.

R $3549240 \quad$ H 5824700

40821 B2/704. Alte Tongrube $2 \mathrm{~km} \mathrm{~W} \mathrm{Ortsmitte}$ Mellendorf, aus 1,5 $\mathrm{m}$ Tiefe.

R $3548040 \quad$ H 5823830

2140822 B1/270. Alte Tongrube $500 \mathrm{~m}$ SW Schadehop, aus $1 \mathrm{~m}$ Tiefe.

R $3545230 \quad$ H 5821790

40823 Alte Tongrube $1,5 \mathrm{~km}$ SE Brelingen, aus $1,5 \mathrm{~m}$ Tiefe.

R 3547540

H 5823960

41437 Bohrung in der Sandgrube in HellendorfMühlenfeld (D 23), aus 24-25 m Tiefe (unter Grubensohle).

R 3549780 H 5825130

2441465 B4/1. Wietze-Niederung, am Trülldamm, aus $1,5-2 \mathrm{~m}$ Tiefe.

R $3555150 \quad$ H 5819430
Rückstand vorwiegend aus Gipskristallen mit Foraminiferen des Unter-Barrême (u. Hauterive?) und vereinzelte des Unter-Alb.

Mittel-Alb.

Mittel-Alb.

(Mittleres) Barrême, mit Pleistozän verunreinigt.

Oberkreide, A $1 \mathrm{~b}$ und Barrême, mit Pleistozän verunreinigt.

Pleistozän mit sandschaligen Foraminiferen aus dem (mittleren) Barrême.

Unter-Alb, mit Pleistozän ver-unreinigt.

Unter-Alb, mit Pleistozän verunreinigt.

Unter-Alb, mit Pleistozän verunreinigt.

Fossilfrei. Petrographie des Rückstandes deutet auf Barrême / Apt.

Einzelne Mikrofossilien weisen Ober-Hauterive und zurücktretend Unter-Alb (vermutlich auch Oberkreide) nach.

Unter-Alb, verunreinigt.

Ober-Apt, mit Pleistozän (und Unter-Alb-Anteilen ?) verunreinigt.

Pleistozän. Im Rückstand keine organischen Reste.

Aus dem Großblatt 61 der Geotektonischen Karte von Nordwestdeutschland 1:100000 (Aldinger u. a. 1946) sowie aus den Untersuchungen von Forche (1949) und Bartenstein (1959) sind die Ausstrichgebiete der einzelnen Stufen der Unterkreide, der Oberkreide und 
das Verbreitungsgebiet des Tertiärs im Untersuchungsgebiet und seiner Umgebung bekannt. Die Süd-Grenze der geschlossenen Tertiärverbreitung läuft in einem weiten Bogen nördlich um das Gebiet des Blattes 3424 Mellendorf der Karte 1:25000 herum, über Burgdorf, Fuhrberg, den Salzstock von Hope und von dort in einem weiten Bogen auf das Steinhuder Meer zu. Diese Grenze ist von Mellendorf in nordöstlicher Richtung etwa $9 \mathrm{~km}$, in nördlicher Richtung etwa $8 \mathrm{~km}$ und in nordwestlicher $12-15 \mathrm{~km}$, teilweise noch etwas weiter, entfernt.

Das Ausstrichgebiet der Oberkreide setzt, in nordöstlicher und nördlicher Richtung gemessen, in etwa $7 \mathrm{~km}$ Entfernung von Mellendorf ein. In nordwestlicher Richtung steht die Oberkreide erst in etwa $20 \mathrm{~km}$ Entfernung an. Das Fehlen von Tertiärschuppen bzw. Schollen in den Mellendorfer und Brelinger Bergen und tertiärer Faunenrelikte in den untersuchten Tonproben spricht dafür, daß das Einzugsgebiet der glaziären Schollen und Schuppen südlich der Verbreitungsgrenze des Tertiärs liegt, daß also ein Ferntransport über mehr als 8-9 km als unwahrscheinlich angesehen werden kann. Die wenigen, nur als Verunreinigung in den untersuchten Tonen gefundenen Mikrofossilien der Oberkreide sprechen zudem dafür, daß das Herkunftsgebiet der Tone in der Umgebung von Mellendorf im wesentlichen auch südlich des Ausstrichgebietes der Oberkreide liegt.

Die Richtung des Transportes ergibt sich aus dem beobachteten Einfallen schräggestellter Schichten und aus der Form und dem Streichen der auskartierten Tonvorkommen. Die Tone aus der Umgebung des Löns-Sees, vom Witrem-Berg und dem Waldgebiet östlich Brelingen, also fast alle westlich der Straße Hannover-Schwarmstedt gelegenen Tonvorkommen, wurden von W bzw. NW herantransportiert. Die Form der Tonvorkommen bei Scherenbostel spricht für einen Transport aus nördlichen Richtungen, die der Tone in der unmittelbaren Umgebung von Mellendorf und Bissendorf für einen Transport aus NE. Die Tone von Bennemühlen und aus den Brelinger Bergen dürften ebenfalls von $\mathrm{N}$ gekommen sein. In den genannten Richtungen stehen, oft gar nicht weit entfernt, die entsprechend alten Schichten an. So findet man anstehendes Barrême $4-5 \mathrm{~km}$ westlich bis nordwestlich des Löns-Sees im Gebiet südlich von Negenborn (Bl. 3423 Otternhagen), anstehendes Alb in 7-8 km Entfernung von den Vorkommen, aus denen die Proben 12, 13 und 22 stammen (s. Abb. 4). Nördlich und nordöstlich Mellendorf steht das Alb weitflächig unter pleistozänen Ablagerungen an, meist sogar unmittelbar im Liegenden der hier auftretenden Tone.

Etwas unklar ist, wo die Tone von Scherenbostel herstammen (z. B. Probe 7), die lediglich Mikrofossilien des Apts und der Oberkreide lieferten und in denen Credner (1865) Fossilien des Barrêmes fand. Nach den Ergebnissen der Kartierung sollten diese Tone ebenfalls aus nördlicher Richtung gekommen sein.

Auf Schwierigkeiten stößt ferner die Deutung des Befundes der Proben 6 und 14, in denen Fossilien des Apts und Albs, daneben solche des Barrêmes, vielleicht auch des Hauterives, nachgewiesen wurden. Die Ergebnisse von Bohrungen auf dem Gelände der Molkerei in Mellendorf und in einer Sandgrube in Hellendorf-Mühlenfeld, die beide noch Apt antrafen (Nr. 23 der Tab. 1), lassen uns nördlich bzw. norwestlich von Mellendorf eine kleine, sich an die N-Flanke des Neustadt-Engelbosteler Sattels anschließende Aufsattelung in den Schichten der Unterkreide vermuten, in der ältere Schichten als die des Albs unter Quartärbedeckung anstehen. Wie weit diese Aufsattelung nach SE reicht und ob hier auch noch Schichten der älteren Unterkreide anstehen, ist bis jetzt nicht bekannt. Hierher können eventuell Tone der Proben 6 und 14 stammen. Der Transportweg wäre in diesem Falle etwa 3-4 km lang. Anderenfalls kämen als Herkunftsgebiet weiter im NE gelegene Salzstöcke in Frage, an deren Flanken ältere Unterkreide unter Quartär ansteht. Da aber in deren Umgebung weit verbreitet Tertiär auftritt, das nach unserer bisherigen Kenntnis hier fehlt, bleibt auch diese Deutung unbefriedigend. 


\section{Stratigraphische und regionale Einordnung}

Die Mellendorfer und die Brelinger Berge gehören zu einem großen Stauch-Endmoränenzug, der sich von der deutsch-niederländischen Grenze bis in die Gegend nördlich Braunschweig verfolgen läßt. Woldstedt $(1928,1955)$ prägte dafür den Ausdruck „Rehburger Stadium" bzw. „Rehburger Stauchendmoräne“. Nach dem Gliederungsvorschlag von LÜтTIG (1959) ist der Kern dieses Endmoränenzuges während der Rehburger Phase entstanden.

Die Untersuchungen von Lütrig (1959) ergaben ferner, daß sich das Drenthe-Stadium hier in 5 Phasen gliedern läßt. Auf die Northeimer und die Gronauer Phase folgt die Rehburger Phase, deren Bildungen dann vom Eis der Hamelner Phase überfahren wurden. Während der jüngsten, der Heisterberg-Phase, drang das Inlandeis nur etwa bis in die Höhe von Nienburg vor.

Richter, SChNeider \& W Untersuchungen in der Stauchzone von Uelsen-Itterbeck bzw. im Gebiet von Bersenbrück das sog. "Rehburger Stadium“ in das frühe Drenthe-Stadium, da es vom Eis des HauptDrenthestadiums ïberfahren wurde und Sander fehlen.

Wie bereits erwähnt, gibt es auch in den Mellendorfer Bergen Anhaltspunkte für mehrere Eisvorstöße. So kann man annehmen, daß die bei Scherenbostel auftretenden gestauchten Tone, die sich nicht ohne Weiteres in eine der vorherrschenden Richtungen einordnen lassen, etwas älter als die übrigen sind. Besonders interessant waren die Befunde in der Sand- und Kiesgrube der Firma KüKER auf dem Siemsberg bei Mellendorf, wo im Jahre 1961 auf der Grubensohle unter etwa 10-12 mächtigen Vorschüttsanden gestauchte bzw. gefaltete Schichten aufgeschlossen waren. Geschiebelehm, etwas blaugrauer Ton und kiesige Sande waren intensiv gefaltet. Die Vergenz der Falten ließ auf einen Eisschub von E schließen. Der eingefaltete Geschiebelehm ist nach seiner relativ hellen, graubraunen Farbe, dem Fehlen der im Geschiebelehm der Elster-Eiszeit immer wieder beobachteten Braunkohlenbröckchen und dem Verhältnis der durchscheinenden zu opaken Feuersteinen $(\mathrm{Fd} / \mathrm{Fu}=1,4: 1 \mathrm{bzw} .1,7: 1)$ als drenthestadial anzusprechen (Ḧ̈LBICH 1962, K. RICHTER 1962). Durch die Nordwand des Aufschlusses zieht sich etwa auf halber Höhe eine 0,5-1 m starke gebankte Geschiebelehmlage. Sie selbst und die sie unmittelbar unterlagernden Sande und Kiese sind ebenfalls gestaucht, und 3 flach nach Osten einfallende Verwerfungen setzen, von hier ausgehend, bis zur Oberfläche durch. Die früher die Vorschüttsande überdeckende Grundmoräne dürfte hier erodiert sein. Dieser Aufschluß läßt 2, vielleicht sogar 3 getrennte Eisvorstöße erkennen. Der älteste ist bezeugt durch den Geschiebelehm auf der Sohle der Grube, der nächstjüngere durch die Stauchungen und Faltungen der im Sommer 1961 wenig über der Grubensohle aufgeschlossenen Schichten. Ob der gebankte Geschiebelehm noch einen dazwischen liegenden weiteren Vorstoß repräsentiert, ist allein auf Grund eines Aufschlusses nicht zu entscheiden. Auch die in der Sand- und Kiesgrube auf dem Kickens-Berg nordwestlich Mellendorf in gestauchten Sanden und Kiesen immer wieder auftretenden Geschiebelehmfetzen weisen auf einen älteren Vorstoß hin. Welchem der vor der Rehburger Phase liegenden Vorstöße dieser Geschiebelehm angehört, läßt sich nicht sagen.

Der Vorstoß, der die Brelinger Berge gestaucht hat, dürfte der Heisterberg-Phase (LüTTIG 1959) entsprechen, deren Stauch-Endmoräne auch hier wieder sehr nahe an die der Rehburger Phase herankommt (Lürtic 1959). Es lassen sich also in den Mellendorfer und Brelinger Bergen 3, vielleicht sogar 4 einzelne Phasen unterscheiden, die Heisterberg-Phase, die Rehburger Phase und eine oder zwei weitere, zeitlich davor liegende Phasen oder Staffeln.

Die Zuordnung der Mellendorfer und der Brelinger Berge zu bestimmten Endmoränenzügen hat öfter gewechselt. SpethmanN (1908) sah in den Brelinger Bergen und dem Siemsberg (zusammen mit dem Kickens-Berg früher als Siemons-Berg bezeichnet) die Fortsetzung 
der Schneerener Endmoräne. Woldstedt (1928) faßte anfänglich die Mellendorfer und Brelinger Berge als die östliche Fortsetzung seines Rehburger Stadiums auf, deutete sie später (WoLDSTEDT 1938, 1939) als eine weniger bedeutende Vorstaffel, kehrte dann aber wieder zu seiner ursprünglichen Ansicht zurück (WoLDSTEDT 1958).

\section{Schrifttum}

Aldinger, H., Closs, H., Schott, W. \& W AGer, R.: Geotektonische Karte von Nordwestdeutschland im Maßstab 1:100000, Großblatt 61. Herausgeb. A. BENTz. - Hannover (Amt Bodenforsch.) 1946.

Bartenstein, H.: Barrême-Scholle von Mellendorf nördlich Hannover, ehemaliger locus typicus von Roemer 1841, Mülleer 1893 und Koenen 1902. - Geol. Jb., 76, S. 453-468, 5 Abb., Hannover 1959.

BeErhenke, F.: Beiträge zur Morphologie und Geologie der Brelinger Berge und des Wietze-Tales nördlich Hannover. - 66 S., 12 Taf., 4 Phot., Ungedr. Dissertation, Hannover 1947.

Beschoren, B.: Geologische Kartierung des Blattes Mellendorf 3424 (1815). (Berlin 1933.) - Archiv. Nieders. Landesamt Bodenforsch. (Reinblatt u. Bohrkarte.)

Credner, H.: Die Verbreitung des Gault in der Umgebung von Hannover. - Z. deutsch. geol. Ges., 17, S. 232, Berlin 1865.

Forche, F.: Der Neustadt-Engelbosteler Sattel. - In: Erdöl und Tektonik in NW-Deutschland, S. 91-95, Hannover-Celle (Amt f. Bodenforsch.) 1949.

Grahle, H.-O. \& Groba, E.: Das Pleistozän des Rehburger Stadiums (Saale-Vereisung) in der weiteren Umgebung von Hannover. - Unveröffentl. Vortr. Arbeitsgem. nordwestdeutsch. Geologen, Eutin/Holstein, 24.-27. 5. 1961.

GrIPP, K.: Glaziologische und geologische Ergebnisse der Hamburgischen Spitzbergen-Expedition 1927..- Abh, naturwiss. Verein Hamburg, 22, H. 3-4, Hamburg 1929.

Нӥцвісн, J.: Gefügeanalytischer Beitrag zur Genese der pleistozänen Rücken zwischen Garbsen und Schillerslage nördlich von Hannover. - Geol. Jb., 80, S. 161-212, 31 Abb., 5, Tab., Hannover 1962.

HAMm, Fr.: Vorläufige Mitteilung über einen als Os gedeuteten Höhenrücken auf dem Nordufer der Leine nordwestlich von Hannover. - Mitt. Provinzialstelle Naturdenkmalpflege, S. 96-100, Hannover 1929.

Keller, G.: Die Beziehung des Rehburger Stadiums südlich Ankum (Kreis Bersenbrück) zur saaleeiszeitlichen Grundmoräne. - Eiszeitalter und Gegenwart 3, S. 58-64, Öhringen/Württ. 1953. - - Drucktexturen in eiszeitlichen Sedimenten. - Eiszeitalter und Gegenwart 4/5, S. 158-171, 9 Abb., Óhringen/Württ. 1954. - - Das Fluvioglazial bei Engelbostel und Frielingen nördlich Hannover. - Geol. Jb. 70, S. 247-260, 5 Abb., 1 Tab., Hannover 1955.

Koenen, A. von: Die Ammonitiden des Norddeutschen Neocom (Valangien, Hauterivien, Barrêmien und Aptien). - Abh. preuß. geol. L.-A., N.F. 24, 451 S., 55. Taf., Berlin 1902.

Köster, R.: Eine neue experimentaltektonische Methode mit Sand-Paraffin-Gemengen. - N. Jb. Geol. Paläont., Mh., 1957, S. 289-295, 3 Abb., Stuttgart 1957 (1957 a). - - Experimente zur glazialen Faltung. - N. Jb. Geol. Paläont., 1957, Mh., S. 510-517, 5 Abb., Stuttgart 1957 (1957 b). - - Experimenteller Beitrag zur Mechanik von Stauchungszonen. Meyniana 6, S. 60-84, 64 Abb., Kiel 1958.

LANG, H. D.: Geol. Karte Niedersachsen $1: 25$ 000, Erl. Bl. Isernhagen Nr. 3524, 130 S., 1 Kt., 5 Faltt., 24 Abb., 6 Tab., Hannover 1962. - - Das Endmoränengebiet der Mellendorfer und Brelinger Berge. - Ber. naturhist. Ges. 107, S. 21-30, 3 Abb., Hannover 1963.

Lürtig, G.: Heisterbergphase und Vollgliederung des Drenthe-Stadiums. - Geol. Jb. 75, S. 419-430, 6 Abb., 1 Tab., Hannover 1959.

MülLER, G.: Über das Vorkommen von Ancyloceras gigas-Schichten bei Mellendorf nördlich Hannover. - Jb. preuß. geol. L.-A. 13 (1892), S. 16-22, Berlin 1893.

Richter, K.: Geschiebekundliche Gliederung der Elster-Eiszeit in Niedersachsen. - Mitt. geol. Staatsinst. Hamburg 31, S. 309-343, 2 Tab., 4 Abb., Hamburg 1962.

Richter, W., Schneider, H. \& W WGER, R.: Die saaleeiszeitliche Stauchzone von Itterbeck-Uelsen (Grafschaft Bentheim). - Z. deutsch. geol. Ges. 102, S. 60-74, Stuttgart 1951.

Roemer, F. A.: Die Versteinerungen des norddeutschen Kreidegebirges. - Hannover 1841.

SpethmanN, H.: Glaziale Stillsandslagen im Gebiet der Weser. - Mitt. geograph. Ges. Lübeck (2) 22, S. 1-17, 1 Abb., Lübeck 1908.

VIETE, G.: Zur Entstehung der glazigenen Lagerungsstörungen unter besonderer Berücksichtigung der Flözdeformationen im mitteldeutschen Raum (mit Beilagemappe). - Freib. Forsch. H., C 78, S. 1-257, 9 Bilder, 30 Taf., 18 Beilagen, 5 Tab., Berlin 1960. 
Woldstedt, P.: Über einen wichtigen Endmoränenzug in Nordwestdeutschland. - Jber. nieders. geol. Ver. 21, S. 10-17, 1 Abb., Hannover 1928. - - Über Vorstoß- und Rückzugsfronten des Inlandeises in Norddeutschland. - Geol. Rdsch. 29, S. 481-490, 2 Abb., Stuttgart 1938. - - Über Endmoränen in der südlichen Lüneburger Heide. - Abh. naturw. Ver. Bremen 31, H. 2, S. 236-246, 3 Abb., Bremen 1939. - - Das Eiszeitalter, 2. Band, Stuttgart 195,8 .

Manuskr. eingeg. 11. 3. 1964.

Anschrift des Verf.: Dr. Hans Dietrich Lang, Niedersächsisches Landesamt für Bodenforschung, 3 Hannover, Wiesenstraße 1. 